\title{
LE KYSTE HYDATIQUE FACIAL ISOLE
}

\author{
M. MEZIANE, H. MIMOUNI, A. OUJILAL, H. BENHALIMA, M. BOULAICH, M. KZADRI \\ SERVICE D'ORL ET DE CHIRURGIE MAXILLO-FACIALE. \\ HÔPITAL DE SPÉCIALITÉ. RABAT. MAROC
}

\begin{abstract}
Le kyste hydatique se présente exceptionnellement comme une masse faciale. Nous rapportons le cas d'un kyste hydatique de la région temporo-jugale chez un patient de 20 ans révélé par une tuméfaction faciale isolée. Le bilan radiologique a montré une formation liquidienne avec pseudo cloisons siégeant au niveau des parties molles temporales gauche. C'est l'exploration chirurgicale qui a posé le diagnostic de kyste hydatique, confirmé par l'étude anatomopathologique. Elle a permis une kystectomie totale. L'évolution a été marquée par l'absence de récidive à 3 ans.
\end{abstract}

Mots clès : Kyste hydatique, face, kystectomie.

\section{SUMMARY}

Hydatid cysts are exceptionally presented as a facial mass. We report a case of hydatid cyst involving in temporo-jugal area in $\mathbf{2 0}$ year old men, revealed by an isolated facial swelling. The radiological assessment showed a liquid formation with pseudo partitions sitting on left temporal soft parts. The surgical exploration allowed a total removal of the cyst and posed the diagnosis of hydatid cyst confirmed by the anatomopathologic examination. The patient showed no evidence of recurrence at his last follow-up, which was 3 years after surgery.

Key words : Hydatid cyst, face, surgical treatment

\section{INTRODUCTION}

Le kyste hydatique sévit en mode endémique au Maroc et constitue un réel problème de santé publique. En dépit de sa fréquence, la localisation faciale de ces kystes reste exceptionnelle, seuls quelques cas ont été rapportés dans le monde. Une tuméfaction faciale isolée fait rarement évoquer un kyste hydatique. Néanmoins, le diagnostic doit être suspecté si une hydatidose diffuse est déjà reconnue.

Nous proposons une observation originale d'un kyste hydatique de la région temporo-jugale dont le seul signe clinique était une tuméfaction temporale.

\section{OBSERVATION}

Mr B.M âgé de 20ans, d'origine rurale et sans antécédents pathologiques notables, a consulté pour une tuméfaction temporo-jugale gauche indolore, évoluant depuis 2ans, augmentant progressivement de volume dans un contexte de conservation de l'état général. L'examen clinique de ce patient a retrouvé une masse temporo-jugale gauche de $3 \mathrm{~cm} / 3 \mathrm{~cm}$, non fistulisée, sans phénomènes inflammatoires en regard, de consistance molle et non douloureuse. L'examen des aires ganglionnaires n'a pas trouvé d'adénopathies satellites. Le reste de l'examen $\mathrm{ORL}$ et Maxillo-faciale était sans particularité. Après un bilan biologique standard qui était normal, un bilan radiologique, fait d'une TDM faciale a été réalisé et a retrouvé une formation ovalaire à composante liquidienne avec des pseudo cloisons siégeant au niveau des parties molles temporales gauches (Figures 1,2).

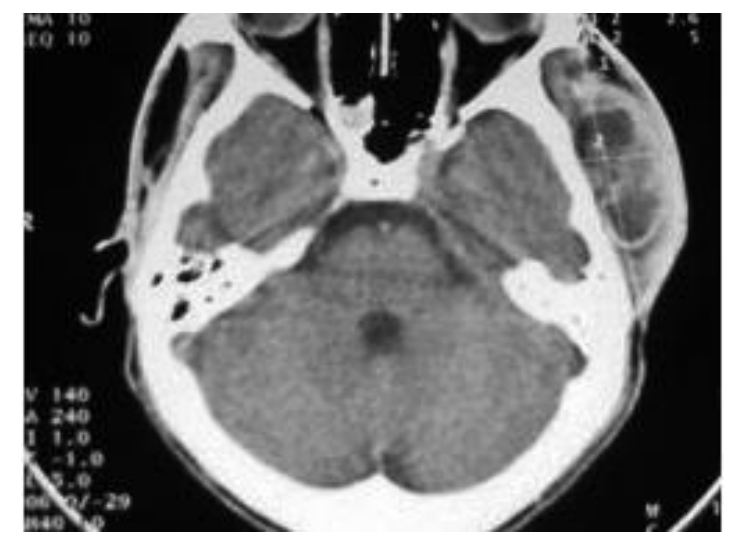

Fig. 1 : TDM en coupe axiale : masse kystique temporale avec pseudocloisons

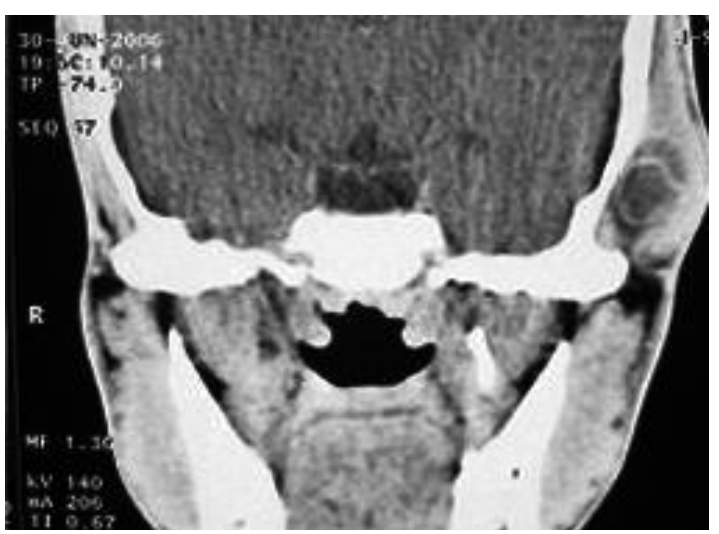

Fig. 1 : TDM en coupe coronale : masse kystique temporale cloisonnée 
Au terme de ces bilans une exploration chirurgicale a été indiquée. Une ponction première du kyste a été effectuée et a ramené un liquide eau de roche. On a réalisé par la suite une kystectomie totale après stérilisation du kyste par de l'eau oxygénée. La pièce opératoire a été adressée au service d'anatomopathologie et la lecture était en faveur d'un kyste hydatique. Les suites opératoires étaient simples.

Dans le cadre du bilan de la maladie hydatique, on a complété par une échographie abdominale et par une radiographie thoracique qui étaient normales.

Un suivi du malade a été assuré avec un recul de trois années et n'a pas montré de récidives ni d'autres localisations.

\section{DISCUSSION}

Les kystes hydatiques sont beaucoup plus fréquents dans le pays où le niveau socioéconomique et sanitaire est bas. La maladie est due à l'infestation par un parasite «'Echinococcus granulosus » dont l'hôte définitif est le chien. L'homme est accidentellement contaminé par ingestion d'œufs du parasite. Une fois dans la circulation, ces œufs se transforme en larves hydatiques qui vont se localiser dans $65 \%$ des cas dans le foie et dans $25 \%$ des cas dans les poumons (1). Les localisations cervico-faciales sont rares, elles représentent $2 \%$ de toutes les localisations (2).

Le siège le plus fréquent est la thyroïde, la parotide et à un degrés moindre la glande sous maxillaire (3). D'autres localisations ont été décrites dans la littérature, au niveau de la mandibule, du sinus maxillaire, de l'orbite, de la fosse infra temporale, de la fosse ptérigopalatine, de l'espace para pharyngé et de la langue.....(4)

Le diagnostic est rarement évoqué devant une tuméfaction faciale isolée, d'autant plus que la symptomatologie est polymorphe et non spécifique. Elle est fonction de la localisation du kyste, sa taille, et sa rapidité d'évolution. Certains patients peuvent présenter un prurit secondaire à leur sensibilité à l'antigène de l'Echinococcus, la rupture peut provoquer une réaction ou choc anaphylactique.

L'anamnèse est importante dans le diagnostic du fait $\mathrm{du}$ polymorphisme des signes cliniques. Elle recherchera l'origine du patient (milieu rural), sa profession (précisera s'il y a notion de contact avec les chiens) et ses antécédents médicochirurgicaux notamment une chirurgie pour un kyste hépatique ou pulmonaire.

\section{REFERENCES}

1. Amr SS, Amr ZS, Jitawi S, Annab H. Hydatosis in Jordan: an epidemiological study of 306 cases. Ann Trop Med Parasitol. 1994; 88:623-7.

2. Bader G, Rose KG, Fohlmeister I. Echinococcus cysticus infections of the head and neck. HNO. 1979; 27:237-41

3. Riquet M, Cohen-Solal G, Soulier A. Kyste hydatique du cou: à propos d'une observation. Ann Otolaryngol Chir Cervicofac. $1982 ; 99: 269-72$.

4. Bouckaert M. R., Raubenheimer J., Jacobs J. Maxillofacial hydatid cysts. Oral Surg Oral Med Oral Pathol Oral Radiol Endod. 2000; 89:338-42

5. Soylu L, Aydogan B, Kiroglu M et al. Hydatic Cyst in the Head and Neck Area. American Journal of Otolaryngology. 199516( 2): 123-5
L'examen clinique notera la localisation exacte du kyste, sa taille, sa consistance, ses rapports anatomiques et recherchera d'autres localisations.

Un complément d'imagerie est nécessaire, il comportera une échographie voire une tomodensitométrie cervicofaciale qui vont permettre d'affirmer la nature liquidienne de la masse, de visualiser des cloisons, de préciser les rapports anatomiques du kyste et d'orienter le diagnostic. La radiographie pulmonaire et l'échographie abdominale à la recherche d'autres localisations sont primordiales.

L'hyper éosinophilie est souvent absente et non spécifique, elle n'est retrouvée que dans $30 \%$ des cas (5). Le diagnostic biologique repose sur le sérodiagnostic basé sur plusieurs techniques, l'immunoélectrophorèse et l'électrosynérèse, méthodes qualitatives, qui s'avèrent les techniques de choix en raison de leur spécificité (6). Quant au test Elisa, il est plus sensible et plus spécifique. Toutefois, les anticorps relevés par ces techniques n'étant pas tous de même nature, il est préférable de coupler les deux techniques. Un certain nombre de kystes restent sérologiquement muets (7). Le traitement de choix du kyste hydatique est la chirurgie. II consiste en une stérilisation première du kyste par injection d'un scolicide (du nitrate d'argent $(0,5 \%)$ ou de sérum salé hypertonique $(20 \%)$ ), suivie d'un traitement du kyste soit par une périkystectomie ou si impossible par une simple résection du dôme saillant.

Le traitement médical ne peut se concevoir que dans certaines situations : une plurikystose, une localisation kystique inaccessible chirurgicalement, chez un patient dont l'état de santé ne peut pas supporté la chirurgie ou pour la prévention des récidives sur rupture du kyste (5). II est à base d'albendazol (10mg/Kg/j) ou mebendazol (50 à100mg/Kg/J) (8). Un suivi doit être assuré pour guetter d'éventuelles récidives, mais le pronostic reste généralement très bon.

\section{CONCLUSION}

La localisation cervico-faciale du kyste hydatique reste rare, même dans las pays d'endémie. Elle doit figurer dans le diagnostic différentiel des tuméfactions cervico-faciales pour permettre un diagnostic précoce et une prise en charge adéquate.

Le traitement de choix est la chirurgie. Mais le meilleur traitement reste prophylactique par rupture du cycle évolutif du parasite. Barahioui M. Dyspnée laryngée révélatrice d'une localisation cervicale d'hydatidose : A propos d'un cas

Arch Pédiatr. $2001 ; 8$ : 1341-3

7. Wattre P, Capron M, Bekhti A, Capron A. Diagnostic immunologique de l'hydatidose : 139 observations. Nouv Presse Med. $1980 ; 9$ : 305-9. 8. Ataoglu H, Uckan S et al. Maxillofacial Hydatid Cyst. J Oral Maxillofac Surg. 2002; 60: 454-6 